\title{
Abkürzungs- und Symbolverzeichnis
}

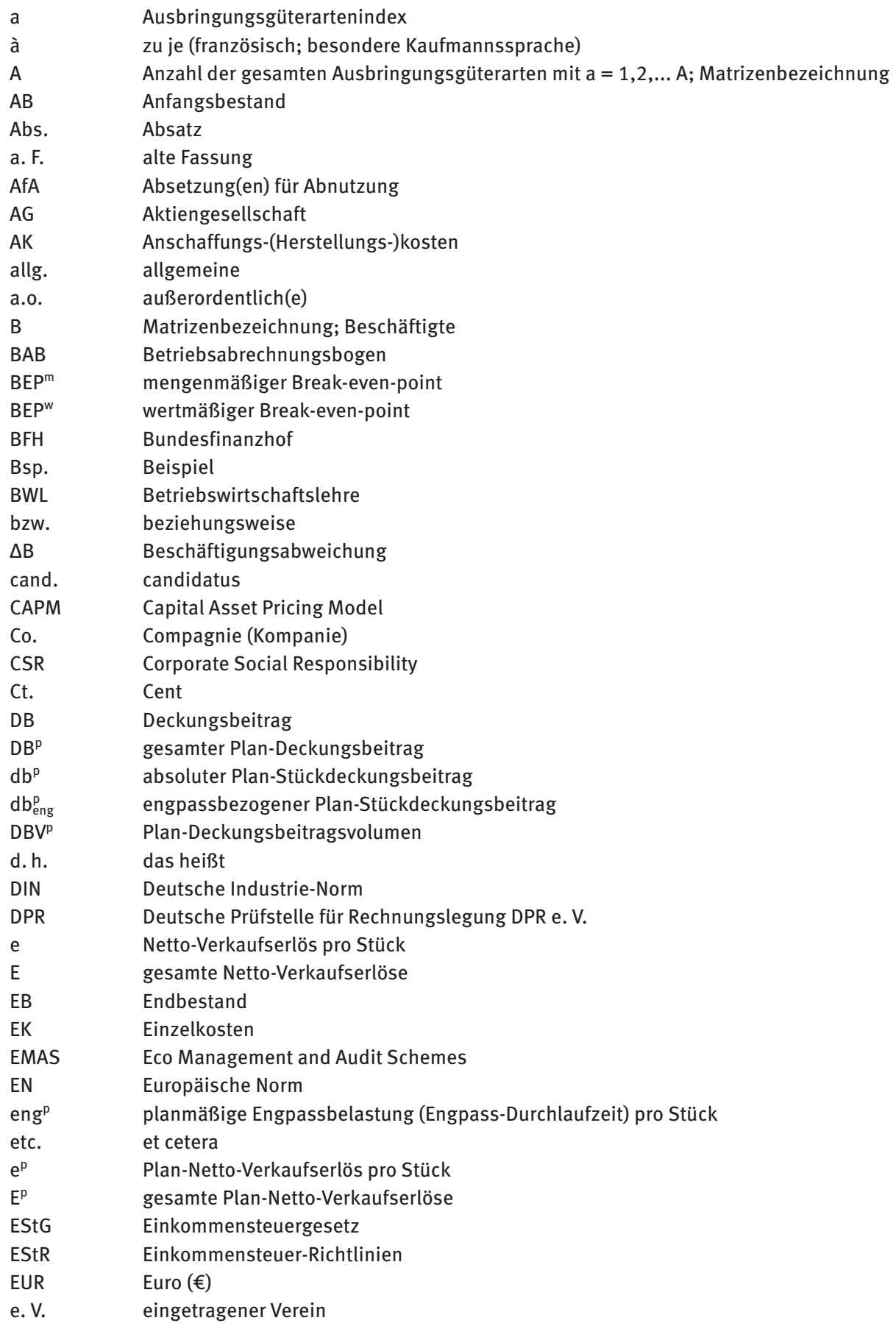




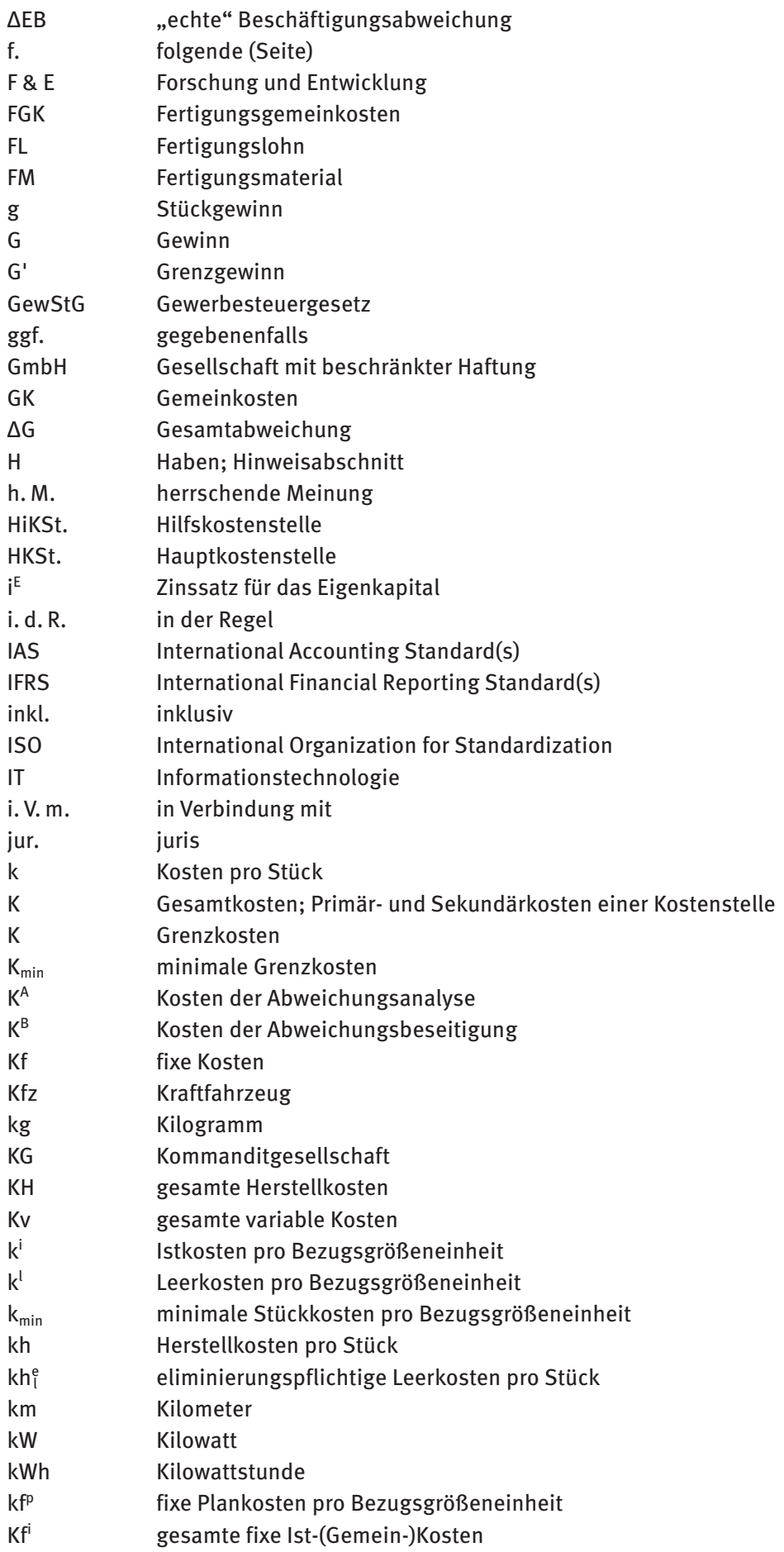


Kf ${ }^{p} \quad$ gesamte fixe Plan-(Gemein-)Kosten

$\mathrm{K}^{\mathrm{i}}\left(\mathrm{x}^{\mathrm{i}}\right) \quad$ gesamte Ist-(Gemein-)Kosten auf der Basis von Istpreisen

$\mathrm{K}^{\mathrm{i}^{*}}\left(\mathrm{x}^{\mathrm{i}}\right) \quad$ gesamte Ist-(Gemein-)Kosten auf der Basis von Planpreisen

$\mathrm{K}^{\mathrm{l}} \quad$ Leerkosten

$\mathrm{K}^{\mathrm{n}} \quad$ Nutzkosten

$\mathrm{KH}_{\mathrm{l}}^{\mathrm{e}} \quad$ gesamte eliminierungspflichtige Leerkosten

KHf gesamte fixe Herstellkosten

ko Opportunitätskosten

KOG Kostenobergrenze eines variablen Einsatzfaktors

KP Primärkosten einer Kostenstelle

$\mathrm{k}^{\mathrm{p}} \quad$ Plankosten pro Bezugsgrößeneinheit

$\mathrm{K}^{\mathrm{p}} \quad$ gesamte Plankosten

$\mathrm{K}_{\mathrm{SW}}^{\mathrm{P}} \quad$ planmäßig anfallende Stilllegungs-, Stillstands- und Wiederanlaufkosten

$K^{p}\left(x^{p}\right) \cdot \frac{x^{i}}{x^{p}} \quad$ verrechnete Plan-(Gemein-)Kosten bei Ist-Beschäftigung

$\mathrm{K}^{\mathrm{P}}\left(\mathrm{x}^{\mathrm{i}}\right) \quad$ gesamte Soll-(Gemein-)Kosten [Plan-(Gemein-)Kosten bei Ist-Beschäftigung]

$\mathrm{K}^{\mathrm{P}}\left(\mathrm{x}^{\mathrm{p}}\right) \quad$ gesamte Plan-(Gemein-)Kosten bei Planbeschäftigung

$\mathrm{Kf}^{\mathrm{P}}\left(\mathrm{x}^{\mathrm{P}}\right) \quad$ gesamte fixe Kosten bei Planbeschäftigung

ks Selbstkosten pro Stück

KStG Körperschaftsteuergesetz

kv variable Kosten pro Stück

$\mathrm{kv}^{\mathrm{p}} \quad$ variable Plankosten pro Stück

$\mathrm{Kv}^{\mathrm{p}} \quad$ gesamte variable Plankosten

$\mathrm{KV}^{\mathrm{P}} \quad$ Plan-(Gemein-)Kostenverrechnungssatz

$\mathrm{Kv}^{\mathrm{P}}\left(\mathrm{x}^{\mathrm{P}}\right) \quad$ gesamte variable Kosten bei Planbeschäftigung

$\mathrm{Kv}^{\mathrm{p}^{*}}\left(\mathrm{x}^{\mathrm{P}}\right) \quad$ gesamte variable Plankosten bei Planbeschäftigung ohne Kostenanteil des zu untersuchenden variablen Einsatzfaktors

$\mathrm{kv}_{\min } \quad$ minimale variable Kosten pro Stück

KWV gesamte Verwaltungs- und Vertriebskosten

$\Delta \mathrm{K} \quad$ gesamte Kostenabweichung

$\Delta \mathrm{Ke} \quad$ Abweichung zwischen Ist- und Plan-Einzelmaterialkosten

$\Delta \mathrm{Kf}_{\mathrm{ab}}^{\mathrm{P}} \quad$ während der Stillstandszeit abbaufähige fixe Plankosten

I Liter

LKW Lastkraftwagen

Imi leistungsmengeninduziert

Imm leistungsmengenneutral

Max! Maximum

ME Mengeneinheiten

MGK Materialgemeinkosten

Mio. $€ \quad$ Millionen Euro

Min. Minute(n)

n Kostenstellenindex

$\mathrm{N} \quad$ Anzahl der gesamten Kostenstellen mit $\mathrm{n}=1,2, \ldots, \mathrm{N}$

OHG offene Handelsgesellschaft

p Preis pro Einsatzgüterart (Mengeneinheit); Verrechnungspreis einer innerbetrieblichen Leistungseinheit

PC Personalcomputer

PKW Personenkraftwagen

$\mathrm{p}^{\mathrm{i}} \quad$ Istpreis pro Mengeneinheit 


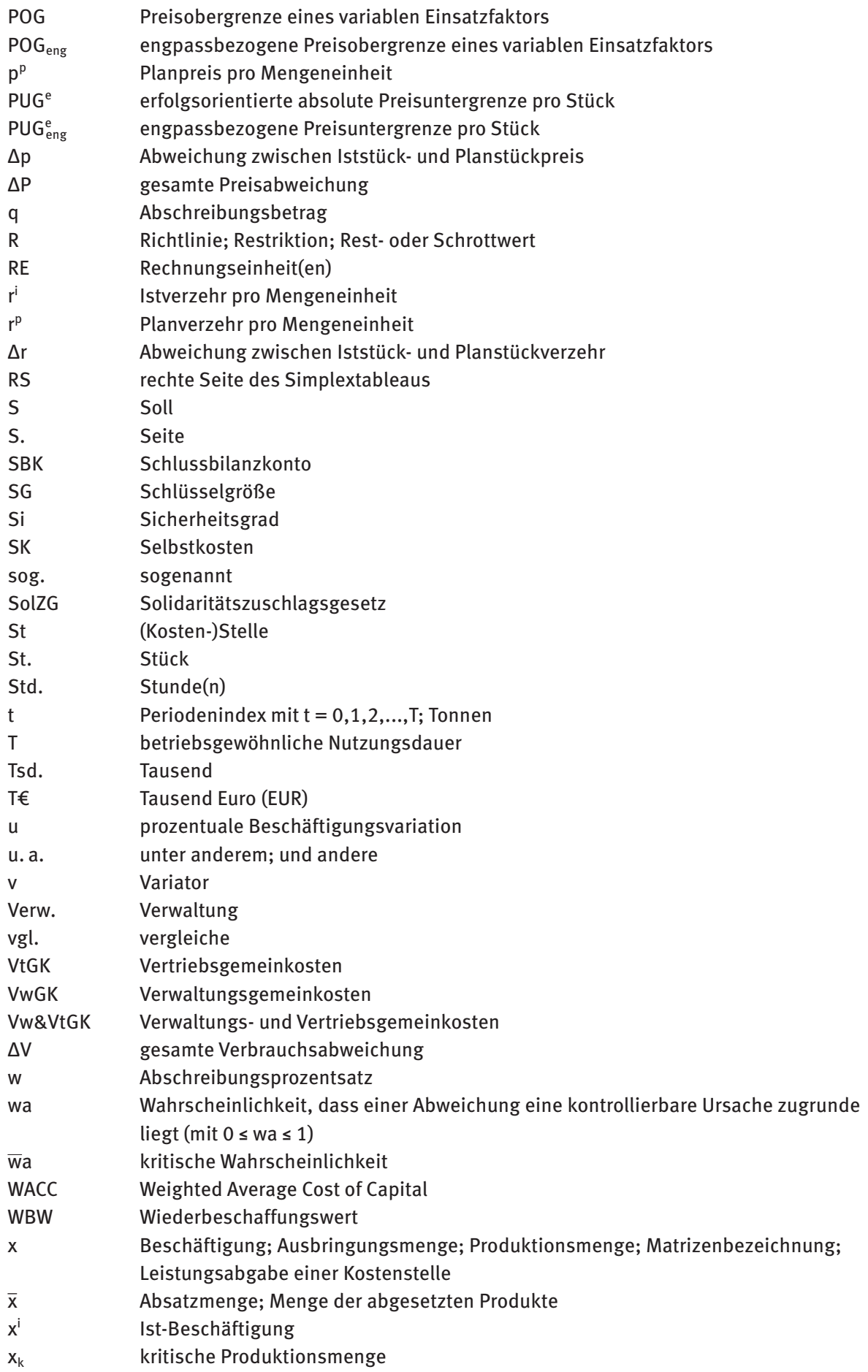




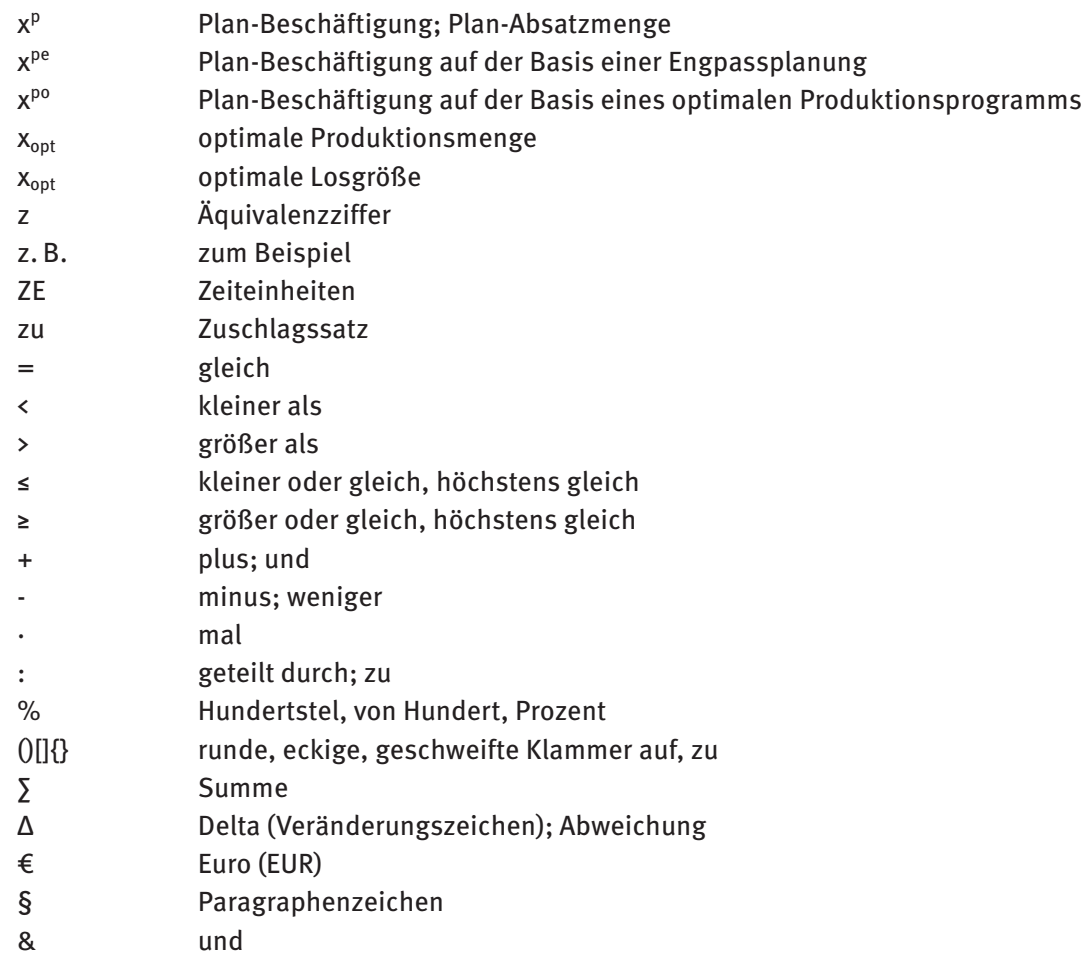


